OPEN ACCESS

Edited by:

Tycho M. Hoogland,

Erasmus MC, Netherlands

Reviewed by:

Richard David Veenstra SUNY Upstate Medical University,

USA

Georg Zoidl,

York University, Canada

${ }^{*}$ Correspondence: Ilse J. Smolders ilse.smolders@vub.ac.be

+'Shared senior authorship.

Received: 29 January 2016 Accepted: 02 December 2016 Published: 20 December 2016

Citation:

Walrave L, Vinken M, Albertini G, De Bundel $D$, Leybaert $L$ and Smolders IJ (2016) Inhibition of Connexin43 Hemichannels Impairs Spatial Short-Term Memory without Affecting Spatial Working Memory.

Front. Cell. Neurosci. 10:288. doi: $10.3389 /$ fncel.2016.00288

\section{Inhibition of Connexin43} Hemichannels Impairs Spatial Short-Term Memory without Affecting Spatial Working Memory

\author{
Laura Walrave ${ }^{1}$, Mathieu Vinken ${ }^{2}$, Giulia Albertini ${ }^{1}$, Dimitri De Bundel ${ }^{1}$, Luc Leybaert ${ }^{3+}$ \\ and IIse J. Smolders ${ }^{1 * t}$ \\ ${ }^{1}$ Department of Pharmaceutical Chemistry, Drug Analysis and Drug Information, Center for Neurosciences, Vrije Universiteit \\ Brussel, Brussels, Belgium, ${ }^{2}$ Department of In Vitro Toxicology and Dermato-Cosmetology, Vrije Universiteit Brussel, \\ Brussels, Belgium, ${ }^{3}$ Physiology Group, Department of Basic Medical Sciences, Ghent University, Ghent, Belgium
}

Astrocytes are active players in higher brain function as they can release gliotransmitters, which are essential for synaptic plasticity. Various mechanisms have been proposed for gliotransmission, including vesicular mechanisms as well as non-vesicular ones, for example by passive diffusion via connexin hemichannels (HCs). We here investigated whether interfering with connexin43 (Cx43) HCs influenced hippocampal spatial memory. We made use of the peptide Gap19 that blocks HCs but not gap junction channels and is specific for $\mathrm{C} \times 43$. To this end, we microinfused transactivator of transcription linked Gap19 (TAT-Gap19) into the brain ventricle of male NMRI mice and assessed spatial memory in a $\mathrm{Y}$ maze. We found that the in vivo blockade of $\mathrm{C} \times 43 \mathrm{HCs}$ did not affect the locomotor activity or spatial working memory in a spontaneous alternation $Y$ maze task. Cx43 blockade did however significantly impair the spatial short-term memory in a delayed spontaneous alternation $Y$ maze task. These results indicate that $\mathrm{Cx} 43 \mathrm{HCs}$ play a role in spatial short-term memory.

Keywords: connexin43, Y maze, TAT-Gap19, working memory, short-term memory

\section{INTRODUCTION}

Over the few last years, evidence has accumulated pointing to a key role for connexin 43 (Cx43) signaling in neurophysiology (Cheung et al., 2014). Cx43 forms gap junctions (GJs) and hemichannels (HCs), which mediate intercellular and extracellular communication, respectively (Chandrasekhar and Bera, 2012). In the central nervous system, $\mathrm{Cx} 43$ is expressed in developing neurons, activated microglia, pericytes and endothelial cells of blood vessels, but it is predominantly and abundantly found in astrocytes (Naus et al., 1991; Nagy and Rash, 2000; Contreras et al., 2004; Chew et al., 2010; Winkler et al., 2011).

While astrocytic $\mathrm{Cx} 43$ GJs connect two adjacent astrocytes and are normally open under physiological circumstances, astrocytic $\mathrm{Cx} 43$ HCs are located between the cytosol of a single astrocyte and its extracellular environment and have long been assumed to be inactive in basal conditions due to their low open probability at resting membrane potential and baseline ionic concentrations (Contreras et al., 2003; Giaume et al., 2013; De Bock et al., 2014). 
Accordingly, astrocytic HCs seem predominantly active in pathological conditions, e.g., ischemia and inflammation, where they are proposed to be involved in damage-associated adenosine triphosphate (ATP) release, disturbed transmembrane ion fluxes and loss of essential metabolites (Giaume et al., 2013; De Bock et al., 2014; Wei et al., 2014; Montero and Orellana, 2015).

Work by Torres et al. (2012) on acute hippocampal slices however demonstrated that lowering the extracellular calcium ion $\left(\mathrm{Ca}^{2+}\right)$ concentration to levels that can reasonably be attained during neuronal burst activity, triggers Cx43-dependent ATP release that subsequently influences the activity of inhibitory neurons. This work suggested that astrocytic Cx43 HC opening, gliotransmitter release and modulation of synaptic signaling might be operational under physiological conditions. In line with this idea, it has been shown that astroglial Cx43 HCs can open under resting conditions and modulate basal synaptic transmission through ATP (Chever et al., 2014a) and glutamate (Chever et al., 2014b) signaling and that deletion of astroglial Cxs alters synaptic transmission and plasticity (Pannasch and Rouach, 2013). Additionally, Stehberg et al. (2012) recently demonstrated that in vivo modulation of $\mathrm{Cx} 43 \mathrm{HCs}$ alters fear memory. For this purpose, they applied the transactivator of transcription linked L2 peptide (TAT-L2), a selective Cx43 HC inhibitor, in the basolateral amygdalae, which blocked fear memory consolidation. Fear learning capacity was recovered after co-infusing a cocktail of gliotransmitters (glutamate, D-serine, glutamine, ATP, lactate and glycine). These experiments substantiate a critical role for in vivo gliotransmission mediated by $\mathrm{Cx} 43 \mathrm{HCs}$ in fear memory. Indeed, it is known that changes in neuronal plasticity, a substrate for memory (e.g., long-term potentiation), can be modulated by astrocytic gliotransmitter release (Ben Achour and Pascual, 2010; Moraga-Amaro et al., 2014).

The aim of our present work is to determine whether $\mathrm{Cx} 43 \mathrm{HCs}$ play a role in spatial memory. To this end, we made use of Gap19, a synthetic nonapeptide derived from the intracellular L2 domain of $\mathrm{Cx} 43$ that specifically inhibits intramolecular cytoplasmic loop (CL)/C-terminal tail interactions, which are essential for Cx43 HCs to open (Wang et al., 2013b; Figure 1). The basis for the HC specificity relates to the fact that CL/Cterminal tail interactions distinctly affect GJs and HCs, with GJs being closed by these interactions while HCs need these interactions to become available to open with electrical or chemical triggers (Iyyathurai et al., 2013; Wang et al., 2013b). By binding to the C-terminal tail, Gap19 prevents CL/Cterminal tail interactions and thereby keeps $\mathrm{Cx} 43 \mathrm{HCs}$ closed and refractive to potential opening triggers (Wang et al., 2013b). The specificity of Gap19 for Cx43 relates to the fact that the target of Gap19 (i.e., the C-terminal tail) is intracellularly located and the intracellular domains of the $\mathrm{Cx}$ proteins are the least conserved regions in contrast to the extracellular domains which are targeted by other $\mathrm{Cx} 43$ mimetic peptides (e.g., Gap26 and Gap27; Wang et al., 2013a).

Astrocytes can be excited by an elevation of the intracellular $\mathrm{Ca}^{2+}$ concentration $\left(\left[\mathrm{Ca}^{2+}\right]_{\mathrm{i}}\right)$, which also triggers opening of HCs. Indeed, $\left[\mathrm{Ca}^{2+}\right]_{\mathrm{i}}$ elevation up to $500 \mathrm{nM}$ triggers the opening of $\mathrm{Cx} 43 \mathrm{HCs}$, while higher concentrations close the channels again (De Vuyst et al., 2009). This high $\left[\mathrm{Ca}^{2+}\right]_{i}$ closure of HCs may act as a brake to prevent excessive, pathological HC opening, suggesting these channels may have physiological roles. Interestingly, Gap19 not only inhibits voltage-gated $\mathrm{Cx} 43 \mathrm{HC}$ opening but also $\left[\mathrm{Ca}^{2+}\right]_{\mathrm{i}}$-elevation induced $\mathrm{Cx} 43 \mathrm{HC}$ opening (Wang et al., 2013a).

In this study, we microinfused transactivator of transcription (TAT)-Gap19 in the murine brain ventricle and tested its effects on spatial working memory and spatial short-term memory in a Y maze. Working memory refers to memory as it is used to plan and carry out behavior (Cowan, 2008). It is defined as the maintenance and controlled manipulation of a limited amount of information before recall (Aben et al., 2012). Short-term memory reflects faculties of the mind that can hold a limited amount of information (chunck capacity limits) in a very accessible temporarily state (duration limits; Cowan, 2008). Our results demonstrate that inhibition of $\mathrm{Cx} 43 \mathrm{HCs}$ with TAT-Gap19 impaired spatial short-term memory without affecting locomotor activity or spatial working memory.

\section{MATERIALS AND METHODS}

\section{Animals}

All experiments were performed on male NMRI mice (Charles River Laboratories, Chatillon-sur-Chalaronne, France) weighing 20-25 g at the time of surgery ( $\sim 4$ weeks old). The animals were housed in a temperature/humidity regulated environment with a 10/14 h light/dark cycle and received food pellets and water ad libitum. The mice habituated 1 week to the animal house and 1 day to the experimental room before being used in experiments. All procedures were carried out in accordance with the National Rules on Animal Experiments and were approved by the Ethical Committee for Animal Experiments of the Faculty of Medicine and Pharmacy of the Vrije Universiteit Brussel, Brussels, Belgium. To the best of our abilities, results were reported in accordance with the ARRIVE guidelines (Kilkenny et al., 2010).

\section{Surgical Implantation of an Intracerebroventricular (i.c.v.) Guide Cannula}

NMRI mice were anesthetized in an induction chamber with $4 \%$ isoflurane (Iso-vet ${ }^{\circledR}, 1000 \mathrm{mg} / \mathrm{g}$ isoflurane, Dechra Veterinary Products, Bladel, Netherlands). After induction, anesthesia was maintained during the entire duration of the surgery by $2.5 \%-3 \%$ isoflurane via a facemask. Ketoprofen $(3 \mathrm{mg} / \mathrm{kg}$, Ketofen ${ }^{\circledR}, 10 \mathrm{mg} / \mathrm{mL}$ ketoprofen, Merial, Toulouse, France) was administered subcutaneously at the beginning of the surgical procedure to prevent post-operative pain and inflammation. A guide cannula ( $3 \mathrm{~mm}, 26 \mathrm{GA}$, Bilaney Consultants, Düsseldorf, Germany) was implanted stereotactically in the left murine brain ventricle, using the following coordinates relative to bregma: $+1 \mathrm{~mm}$ medial-lateral, $-0.34 \mathrm{~mm}$ anterior-posterior and $-2.20 \mathrm{~mm}$ ventral-dorsal.

After surgery, the mice received $1 \mathrm{~mL}$ saline $(0.9 \% \mathrm{NaCl}$, Baxter, Lessines, Belgium) intraperitoneally and were placed in 


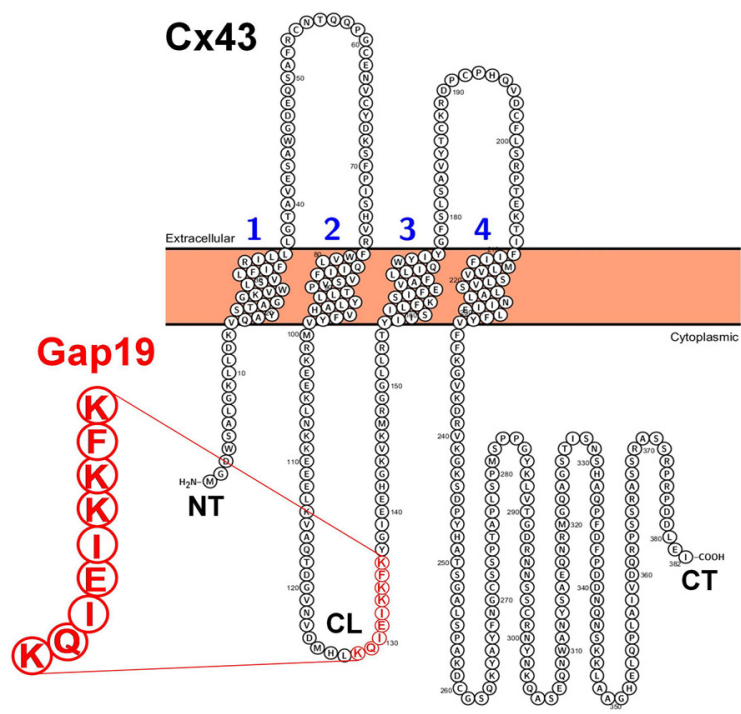

B

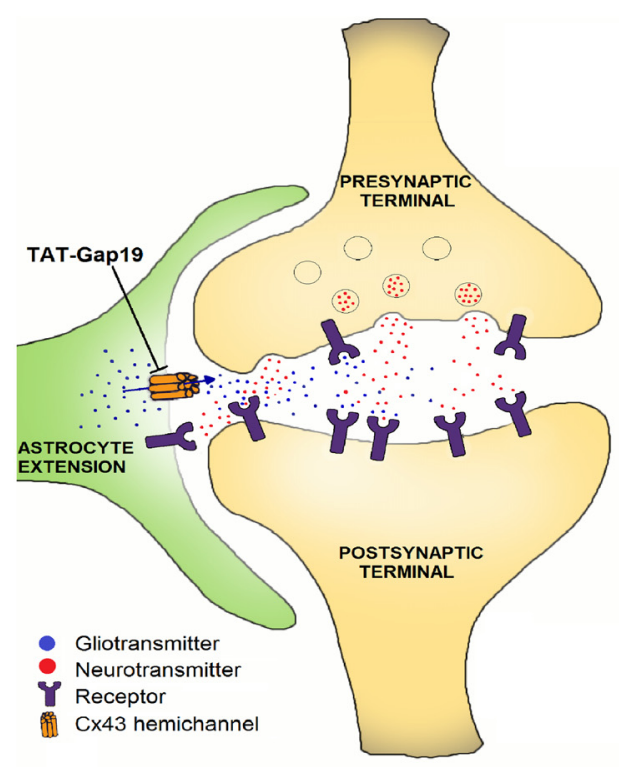

FIGURE 1 | (A) Position of the Gap19 sequence (red) in the cytoplasmic loop (CL) domain of connexin43 (Cx43). Gap19 interacts with the last nine amino acids of the carboxyl terminus (CT) tail, and thereby inhibits Cx43 hemichannel (HC) opening (Illustration generated with the Protter tool (Omasits et al., 2014)) and (B) the tripartite synapse (presynaptic neuron - postsynaptic neuron - astrocyte) in which astrocytic Cx43 hemichannels (HCs) may contribute by releasing neuroactive substances (gliotransmitters), that act as a feedforward signal generated from distant neurons/glial cells or that act in feedback to the synapse upon astrocyte activation by neurotransmitters. $\mathrm{HC}$ opening is inhibited by the selective $\mathrm{Cx} 43 \mathrm{HC}$ inhibitor, transactivator of transcription (TAT)-Gap19.

front of an infrared lamp for 15 min to raise the core body temperature. They were single-housed to prevent cannulated mice from chewing on each other's cannulas and were allowed to recover for 7 days. A stylus (Bilaney Consultants) was placed inside the guide cannula to prevent clogging.

At the end of the experiments, the mice were sacrificed using an overdose of sodium pentobarbital $\left(200 \mathrm{mg} / \mathrm{kg}\right.$, Nembutal ${ }^{\circledR}$, Ceva Santé Animale, Libourne, France) and accuracy of guide implantation was verified postmortem and compared against a mouse brain atlas (Paxinos and Franklin, 2004). A representative image is depicted in Figure 2.

\section{Drugs Used and Their Administration}

We used Gap19 coupled to the TAT membrane translocation motif in order to increase its cell membrane permeability, as the C-terminal tail is intracellularly located (Abudara et al., 2014). TAT-Gap19I130A, an I130A-modified Gap19 analog, was chosen as a negative control peptide since amino acid I130 is involved in the formation of hydrogen bonds and thereby important for Gap19 activity (Wang et al., 2013b). Hence, TAT-Gap19I130A exerts no inhibitory effects on Cx43 HCs (Wang et al., 2013b). Scopolamine, a cholinergic M1 (muscarinic) receptor antagonist, which is often used to generate deficits of performance in the Y maze and other memory tests, was used as a positive control (Kwon et al., 2010; Ohba et al., 2015). Biotin-labeled TAT-Gap19 (biotin-TAT-Gap19) was used to determine peptide distribution following i.c.v. injection.
TAT-Gap19 (MW 2703.28 Da), biotin-TAT-Gap19 (MW 2929.61 Da) and the inactive mutant version TAT-Gap19I130A were synthesized by Pepnome Inc. (Hong Kong, China, $>95 \%$ purity) and dissolved in phosphate buffered saline (PBS; SigmaAldrich, Steinheim, Germany), at a final concentration of $1 \mathrm{mM}$. Scopolamine hydrobromide trihydrate was supplied by SigmaAldrich and dissolved in PBS to yield a final concentration of $33 \mathrm{mM}$. On the day of the experiment, the stylus was removed from the guide cannula and a 33 GA injection cannula (Bilaney Consultants) was inserted through the guide cannula, extending $1 \mathrm{~mm}$ beyond its tip in the ventricle. Compounds were i.c.v. infused via the injection cannula for $2 \mathrm{~min}$ at a flow rate of $0.5 \mu \mathrm{L} / \mathrm{min}$ (total $1 \mu \mathrm{L}$ ), driven by a microinjection pump (CMA 400 Syringe pump, CMA/Microdialysis $\mathrm{AB}$ ), $60 \mathrm{~min}$ prior to testing in the $\mathrm{Y}$ maze. Following drug infusion, injection cannulas were left in place for $1 \mathrm{~min}$ to allow drug diffusion away from the cannula tip. Doses were based on literature (e.g., Stehberg et al., 2012; Abbasi et al., 2013; Tabari et al., 2016). For TAT-Gap19 and TAT-Gap19I130A, mice received $1 \mathrm{nmol} / \mu \mathrm{L}$ or $2.7 \mu \mathrm{g} / \mu \mathrm{L}$ i.c.v. For scopolamine hydrobromide trihydrate, mice received $33 \mathrm{nmol} / \mu \mathrm{L}$ or $10 \mu \mathrm{g} / \mu \mathrm{L}$ scopolamine i.c.v.

\section{Validated Paradigms for Testing Spatial Working and Short-Term Memory in a $Y$ Maze}

Behavioral testing was performed in a $\mathrm{Y}$ maze with three identical, opaque arms at $120^{\circ}$ angle from each other, as 
A

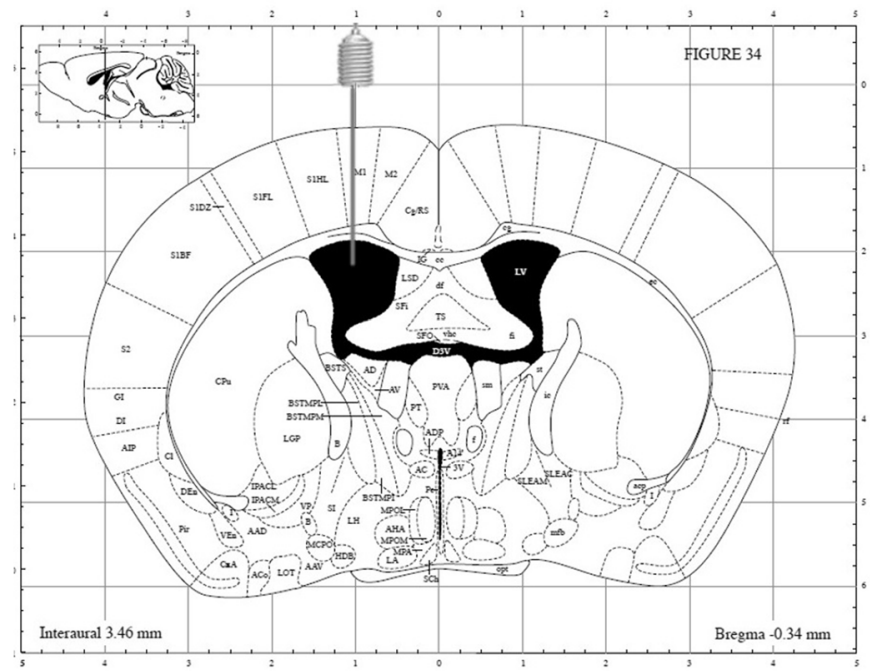

B

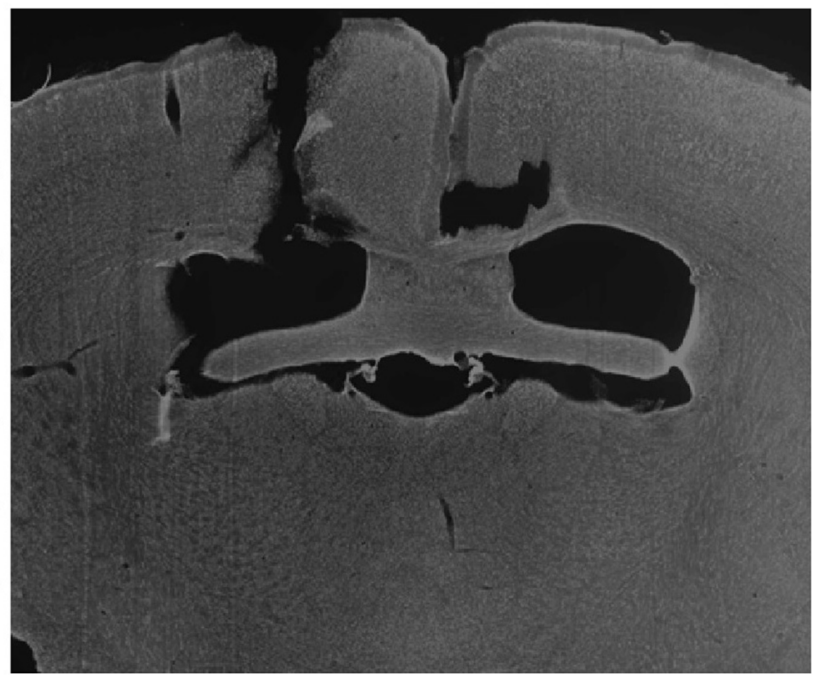

FIGURE 2 | (A) Schematic overview of i.c.v. guide implantation with following coordinates relative to bregma: $+1 \mathrm{~mm} m e d i a l-l a t e r a l,-0.34$ mm anterior-posterior and $-2.20 \mathrm{~mm}$ ventral-dorsal (Paxinos and Franklin, 2004) and (B) representative image of a DAPI counterstained coronal brain section showing accuracy of guide implantation in the left ventricle.

previously described (De Bundel et al., 2011). The arms were $35 \mathrm{~cm}$ long, $5 \mathrm{~cm}$ wide and $8 \mathrm{~cm}$ high, allowing the mice to see distal spatial cues. The Y maze was swabbed with $70 \%$ ethanol between each test to eliminate odors. Solutions were prepared and syringes were filled by one experimenter. Another experimenter encoded the syringes with a number and animals were randomized between these numbers. At the time of data processing and data analyses, the experimenter was blinded to the treatment group.

The continuous spontaneous alternation testing is based on the natural tendency of rodents to explore a novel environment. The mice were placed in the Y maze, facing the wall of one randomly chosen arm and were allowed to freely explore the three arms of the $\mathrm{Y}$ maze for $8 \mathrm{~min}$. Typically, mice explore the least recently visited arm and tend to alternate between the three arms. For efficient alternation, mice rely on their working memory (Wall and Messier, 2002). Every arm entry was recorded manually in order to calculate the percentage of alternation. An entry occurred when all four limbs were within the arm. The total number of arm entries was used as a measure for locomotor activity, while the spontaneous alternation percentage (SAP) was used as a measure of spatial working memory. To calculate the SAP, the total number of alternations (i.e., every time a mouse explored the three arms consecutively) was divided by the total possible alternations (i.e., the number of arm entries minus 2) and multiplied by 100 . Immediate reentries were discounted.

In the delayed spontaneous alternation testing, one of the three arms was closed and the mice were allowed to explore the other two arms for 15 min (training phase). Next, the mice were transferred to their holding cage for $5 \mathrm{~min}$. Subsequently, they were placed into the start arm and were allowed to explore all three arms of the maze for $5 \mathrm{~min}$ (test phase). During these $5 \mathrm{~min}$, the time spent in each arm was recorded manually and the time spent in the novel arm (i.e., previously closed during the training phase) was used as a measure for spatial short-term memory.

\section{Diffusion of Biotin-Labeled TAT-Gap19 into the Mouse Brain}

Peptide diffusion following i.c.v. delivery was determined by injecting $1 \mu \mathrm{L}$ of $1 \mathrm{mM}$ biotin-TAT-Gap19 $(1 \mathrm{nmol} / \mu \mathrm{L}$ or $2.9 \mu \mathrm{g} / \mu \mathrm{L}$ ) or $1 \mu \mathrm{L}$ PBS (control). Immunohistochemistry was performed $60 \mathrm{~min}$ after the injection, in accordance with the in vivo protocol.

Briefly, anesthetized mice $(200 \mathrm{mg} / \mathrm{kg}$ sodium pentobarbital i.p., Nembutal ${ }^{\circledR}$ ) were intracardially perfused for 2 min with $0.9 \% \mathrm{NaCl}$ and $3 \mathrm{~min}$ with $4 \%$ paraformaldehyde (SigmaAldrich) in PBS. Following perfusion, the brains were removed, post-fixed in $4 \%$ paraformaldehyde and sliced into $40 \mu \mathrm{m}$ coronal sections using a vibratome (Leica VT1000S, Leica Biosystems, Diegem, Belgium).

All rinsing steps and incubations were performed in Tris-buffered saline with Triton-X-100 (TBST; $0.01 \mathrm{M}$ Tris, $0.1 \%$ Triton-X-100, $0.9 \% \mathrm{NaCl}, \mathrm{pH} 7.4$; all supplied by SigmaAldrich) at room temperature and under gentle agitation, unless stated otherwise. Free-floating sections were washed with TBST (three times $10 \mathrm{~min}$ ) and blocked with $10 \%$ pre-immune goat (PIG) serum (Milllipore, Temecula, CA, USA) in TBST for $45 \mathrm{~min}$. The sections were then incubated with primary polyclonal rabbit anti-GFAP antibody (diluted 1:1000 in 10\% PIG in TBST, cat. No. Z0334, Dako, Glostrup, Denmark) overnight at $4^{\circ} \mathrm{C}$. The next day, sections were washed with TBST (three times $10 \mathrm{~min}$ ) and incubated with secondary goat anti-rabbit IgG linked to Alexa Fluor 488 (diluted 1:500 in TBST, 
cat. No. 111-545-003, Jackson ImmunoReserach Laboratories, Inc., PA, USA) and streptavidin-labeled CY3 (diluted 1:20 in TBST, Cat. No 434315, Invitrogen, Life Technologies, Ghent, Belgium). Following two washing steps with TBST (each $10 \mathrm{~min})$, nuclei were counterstained with DAPI $(1 \mu \mathrm{g} / \mathrm{mL}$ in TBST, Cat. No. D3571, Life Technologies, Ghent, Belgium) for $5 \mathrm{~min}$ and finally washed with Tris-buffer $(0.01 \mathrm{M}$ Tris, $\mathrm{pH}$ 7.4; two times $10 \mathrm{~min}$ ) before mounting on $\mathrm{VWR}^{\circledR}$ Superfrost ${ }^{\circledR}$ Plus Micro slides (VWR International, Leuven, Belgium).

Slides were coverslipped with DPX mounting medium (Sigma-Aldrich) and images were acquired with a Zeiss LSM710 NLO TiSa multiphoton confocal microscope (excitation wavelength lasers: $488 \mathrm{~nm}, 561 \mathrm{~nm}$ and $790 \mathrm{~nm}$ ), using Zeiss Zen2011 software (Carl Zeiss NV-SA, Zaventem, Belgium).

\section{Data Analysis and Statistics}

Statistical analyses were performed using GraphPad Prism 6.01 with $\alpha$ set at 0.05 . The results are expressed as means \pm standard error of the mean (SEM). All values were normally distributed (D'Agostino and Pearson omnibus normality test) and a two-sided $t$-test with Welch correction was used to denote any differences between experimental groups.

\section{RESULTS}

\section{TAT-Gap19 Does Not Influence Spatial Working Memory or Locomotor Activity}

We first determined whether i.c.v. administered compounds could reliably affect working memory as measured with our Y maze protocol. This validated protocol measures activity/exploratory motivation and spatial working memory, by analyzing the total number of arm entries and the total percentage of correct spontaneous alternations (SAP), respectively. The cholinergic M1 (muscarinic) receptor antagonist was used to generate deficits of performance in the Y maze (de Bruin and Pouzet, 2006; Kwon et al., 2010; Busquet et al., 2012; Ohba et al., 2015).

All animals showed good ambulatory activity (i.e., above six arm entries). We found that i.c.v. administered scopolamine $(10 \mu \mathrm{g} / \mu \mathrm{L})$ significantly decreased the amount of correct alternations (SAP) from 66.54\% \pm 3.25 in control (PBS) to $47.97 \% \pm 2.03$ (both $n=11 ; p=0.0002$; Figure 3A). Scopolamine also increased the total number of arm entries from $35.27 \pm 1.94$ in control animals (PBS) to $65.82 \pm 5.04$ (both $n=11 ; p<0.0001$; Figure 3B).

We next tested TAT-Gap19 $(2.7 \mu \mathrm{g} / \mu \mathrm{L}$ or $1 \mathrm{nmol} / \mu \mathrm{L})$ as well as TAT-Gap19I130A (same concentration), which is composed of an inactive sequence, in the $\mathrm{Y}$ maze protocol. No significant differences were observed in SAP between the TAT-Gap19 treated group $(72.92 \% \pm 2.75)$ compared to the TAT-Gap19I130A treated group $(67.90 \% \pm 1.93$; both $n=16, p=0.2028$; Figure $3 \mathrm{C}$ ) and the exploration rates were not affected as the total arm entries in these experimental groups were not significantly different (TAT-
Gap19I130A: $35.31 \pm 1.73$; TAT-Gap19: $31.94 \pm 1.45$; both $n=16, p=0.1459$; Figure 3D). These results indicate that TAT-Gap19 does not influence locomotor activity or exploratory motivation and that the peptide does not impair spatial working memory.

\section{TAT-Gap19 Impairs the Spatial Short-Term Memory}

Next, we used the well-established delayed Y maze protocol to determine effects at the level of short-term spatial memory (Figure 4). I.c.v. administered scopolamine reduced the time spent in the novel arm to $37.08 \% \pm 2.70(n=8)$ compared to control (PBS) animals $(53.37 \% \pm 4.64$; $n=9 ; p=0.0099$ ), validating that i.c.v. administered scopolamine provokes impaired short-term spatial memory in the delayed Y maze protocol (Figure 4A). Next, we tested TAT-Gap19 and found it to significantly reduce the time spent in the novel arm to $42.60 \% \pm 2.94(n=14)$, compared to TAT-Gap19I130A $(52.14 \% \pm 2.98 ; n=12$, $p=0.0320)$, indicating impaired spatial short-term memory (Figure 4B).

\section{Biotin-TAT-Gap19 Reaches the Hippocampus Following i.c.v. Injection}

Following injection of biotin-TAT-Gap19 in the left lateral ventricle, the peptide was detected by using the biotinstreptavidin method. Positive biotin immunoreactivity was observed in the tissue around the left and right lateral ventricle and around the dorsal third ventricle (ventricular zone; data not shown). Additionally, diffuse labeling of biotin-TAT-Gap19 was observed in the hippocampus (Figures 5B1,2), compared to controls only receiving PBS (Figure 5A).

\section{DISCUSSION}

In the present study, we used a spontaneous alternation and delayed spontaneous alternation $\mathrm{Y}$ maze test in order to investigate the role of $\mathrm{Cx} 43 \mathrm{HCs}$ in spatial working and spatial short-term memory respectively. The Y maze was chosen because of its simplicity and sensitivity to evaluate spatial memory in mice. The alternation principle is based on the exploratory behavior. Mice prefer to visit the less recently visited arm, implicating they need to remember the last arm they visited (Sharma et al., 2010).

The Cx43 mimetic peptide TAT-Gap19 is one of the sole currently available tools to discriminate $\mathrm{Cx} 43$ GJs and HCs. The applied dose of TAT-Gap19 is comparable to the dose of TAT-L2 used by Stehberg and team (Stehberg et al., 2012). In that study, TAT-L2 was micro-infused in both basolateral amygdalae (total $0.5 \mathrm{nmol}$ ) to study the role of $\mathrm{Cx} 43 \mathrm{HCs}$ in fear memory, which is mainly amygdala-dependent. Due to diffusion and dilution effects from the ventricle to other brain regions (e.g., hippocampi), we administered 1 nmol TAT-Gap19. When distributed in an estimated 35-40 $\mu \mathrm{L}$ cerebrospinal fluid volume (Pardridge, 1991; Johanson et al., 2008), this 


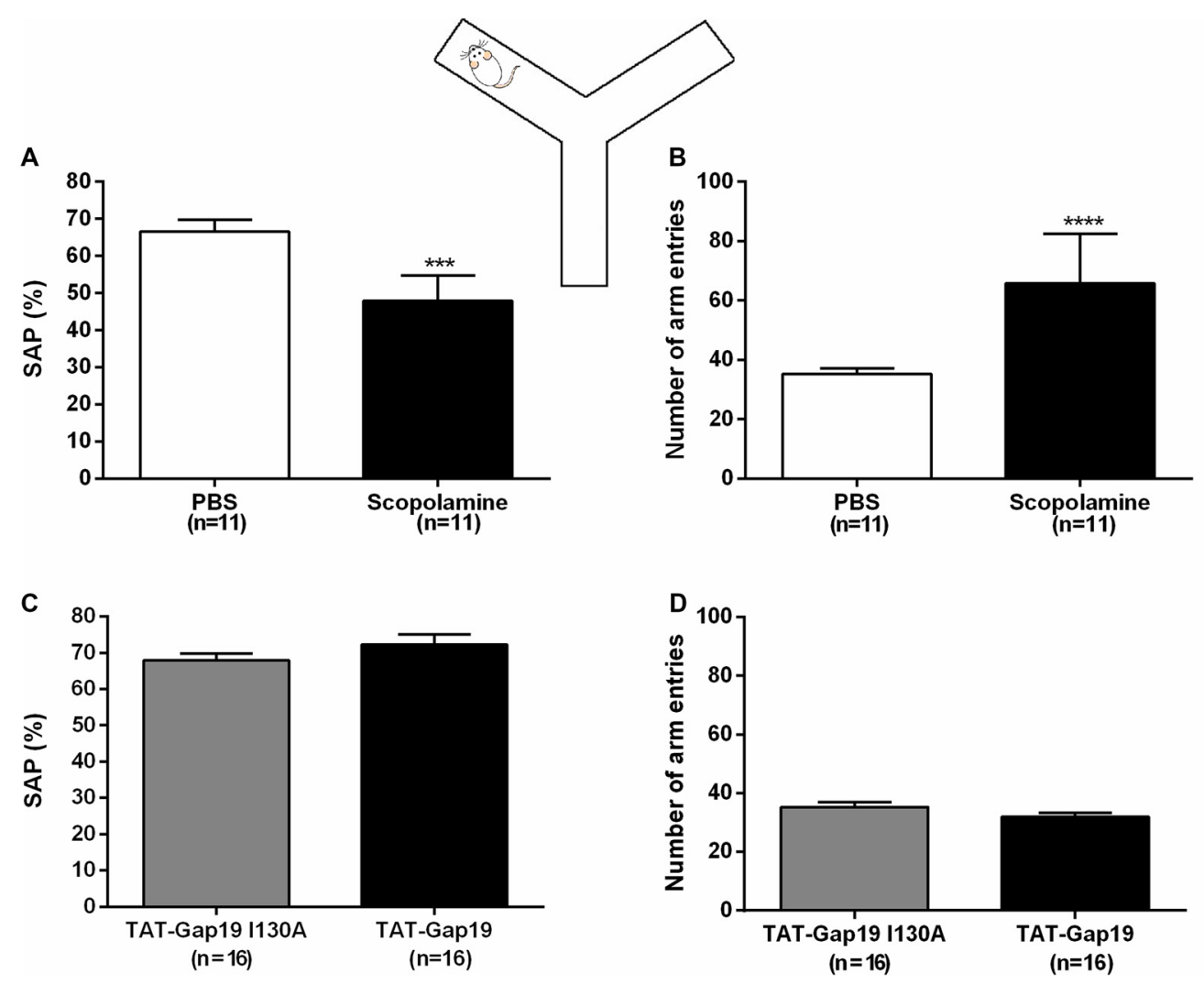

FIGURE 3 | Spontaneous alternation Y maze protocol. Effects of i.c.v. administered scopolamine (10 $\mu \mathrm{g} / \mu \mathrm{L})$ on (A) spontaneous alternation and (B) number of arm entries in the $Y$ maze, compared to vehicle control (PBS). Effects of i.c.v. administered TAT-Gap19 (2.7 $\mu \mathrm{g} / \mu \mathrm{L})$ on (C) spontaneous alternation and (B) number of total arm entries in the $Y$ maze task, compared to the inactive control peptide TAT-Gap19l130A (2.7 $\mu \mathrm{g} / \mu \mathrm{L})$. While scopolamine induced an impairment in spatial working memory $(p=0.0002)$ and an increase in locomotor activity $(p<0.0001)$, TAT-Gap19 did not alter these parameters in the $Y$ maze. Bars represent (A,C) spontaneous alternation percentages (SAP) \pm SEM and (B,D) total number of arm entries \pm SEM. Two-sided $t$-test. ${ }^{* * *} p<0.001$ and $* * * * p<0.0001$.

gives an expected $29-25 \mu \mathrm{M}$ concentration (i.e., four times the half-maximal concentration (i.e., $7 \mu \mathrm{M}$, Abudara et al., 2014)).

Both experimental protocols (spontaneous and delayed spontaneous alternation) were validated with i.c.v. administered vehicle (PBS) and scopolamine (de Bruin and Pouzet, 2006; Kwon et al., 2010; Busquet et al., 2012; Ohba et al., 2015). Scopolamine was used as a positive control as different studies report spatial memory impairments following i.c.v. injection of scopolamine (Abbasi et al., 2013; Tabari et al., 2016). In line with literature, we observed impairments in cognitive function with scopolamine in both experimental protocols, implicating that the $\mathrm{Y}$ maze protocol is sensitive to impairments after i.c.v. delivery of compounds. We also demonstrated an increase in exploratory behavior following i.c.v. scopolamine injection, which complies with a previously published report stating that scopolamine can induce non-cognitive effects, such as increased locomotor activity and hyperactivity (Mathur et al., 1997). Nevertheless, it should be emphasized that the effect of scopolamine does not directly correlate with gliotransmission as scopolamine is a cholinergic M1 (muscarinic) receptor antagonist.
Next, we tested TAT-Gap19 in both set-ups. In the spontaneous alternation test, TAT-Gap19 did not lead to locomotor impairments or deficits in the spatial working memory compared to its inactive mutant version TATGap19I130A. Likewise, Stehberg et al. (2012) observed no impairment in locomotor activity following TAT-L2 injection. In the delayed version of the Y maze, TAT-Gap19 significantly decreased the spatial short-term memory, since we observed a decrease in time spent in the novel arm of about $10 \%$. This decline was in the same order of magnitude as the decline induced by scopolamine $(\sim 16 \%)$. On the contrary, Stehberg et al. (2012) found no impairment in short-term memory due to Cx43 HC inhibition by TAT-L2. However, this research group assessed short-term memory as an increase in freezing behavior in a fear memory paradigm which is mostly amygdala-dependent and not regulated by the dorsal hippocampus, unlike spatial memory.

By injecting biotin-TAT-Gap19, we observed peptide diffusion in the Cornu Ammonis 3 (CA3) region of the dorsal hippocampus. Distribution of biotin-TAT-Gap19 and TAT-Gap19 are presumed to be similar due to the small difference in molecular weight between these peptides 

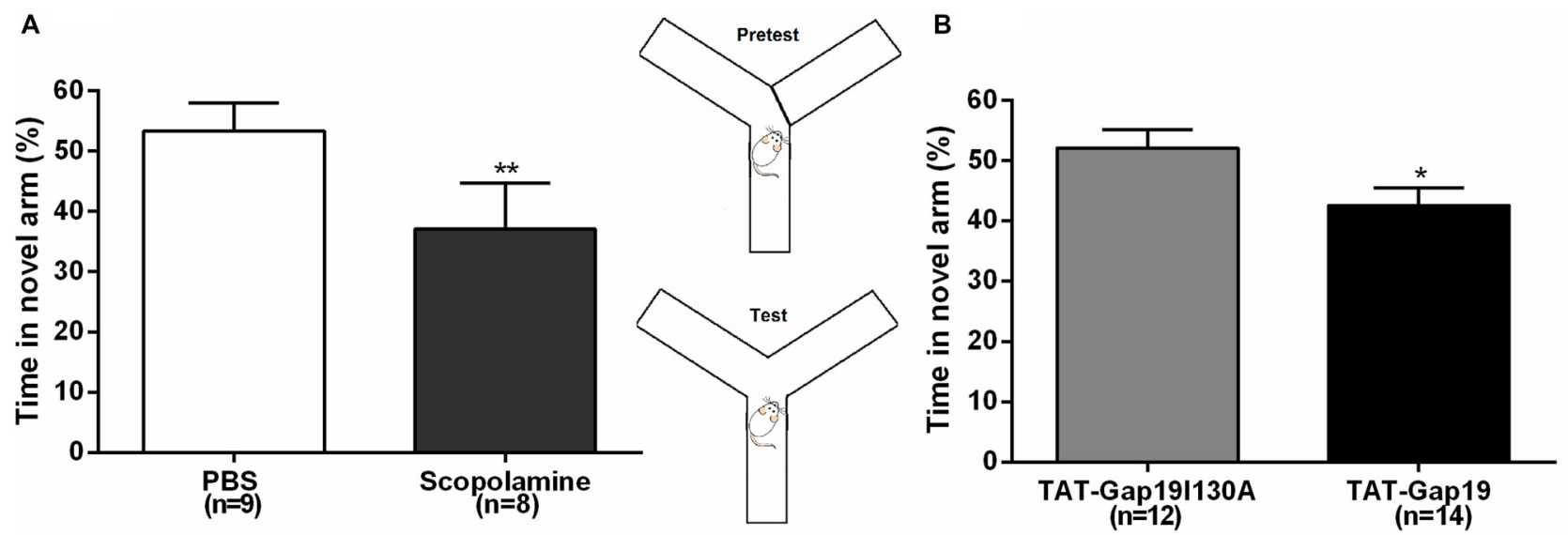

FIGURE 4 | Delayed spontaneous alternation Y maze protocol. Effects of i.c.v. (A) scopolamine (10 $\mu \mathrm{g} / \mu \mathrm{L})$ compared to vehicle control (PBS) and (B) TAT-Gap19 $(2.7 \mu \mathrm{g} / \mu \mathrm{L})$ compared to the inactive control peptide TAT-Gap19l130A $(2.7 \mu \mathrm{g} / \mu \mathrm{L})$ on the time spent in the novel arm. Short-term spatial memory was significantly impaired by scopolamine $(p=0.0099)$ and TAT-Gap19 $(p=0.0320)$. Bars represent percentages (i.e., total time in the novel arm, divided by the total duration of the test (5 min), multiplied by 100) \pm SEM. Two-sided $t$-test; ${ }^{*} p<0.05,{ }^{* *} p<0.01$.

(2929.61 vs. $2703.28 \mathrm{Da})$. As it is known that the dorsal hippocampus (e.g., CA3) is essential once the critical time window requires spatial memory for a longer time period (i.e., 5 min; Lee and kesner, 2003; Yoon et al., 2008; Shipton et al., 2014), it is not surprising that TAT-Gap19 impairs spatial short-term memory. Indeed, as discussed further, hippocampal astrocytes mediate spatial memory through the release of gliotransmitters (Hassanpoor et al., 2014). TAT-Gap19 presumably does not affect spatial working memory since the hippocampus and medial prefrontal cortex jointly contribute to working memory/cognition (Moser et al., 2015) and other associational sensory cortices than the dorsal hippocampus could still provide information on sensory cues to the medial prefrontal cortex (Lee and kesner, 2003). Furthermore, in the hippocampus, the structured network of place cells and grid cells (Moser et al., 2015), which is crucial for spatial memory formation, might be more vulnerable for gliotransmitter modulation (Hassanpoor et al., 2014).

A limitation of this study is that immunoreactivity for biotinTAT-Gap19 could not be demonstrated exclusively in astrocytes. Several biotin-positive cells are apparently not GFAP-positive. However, while $\mathrm{Cx} 43$ is identified in various central nervous system cell types (i.e., developing neurons, activated microglia,
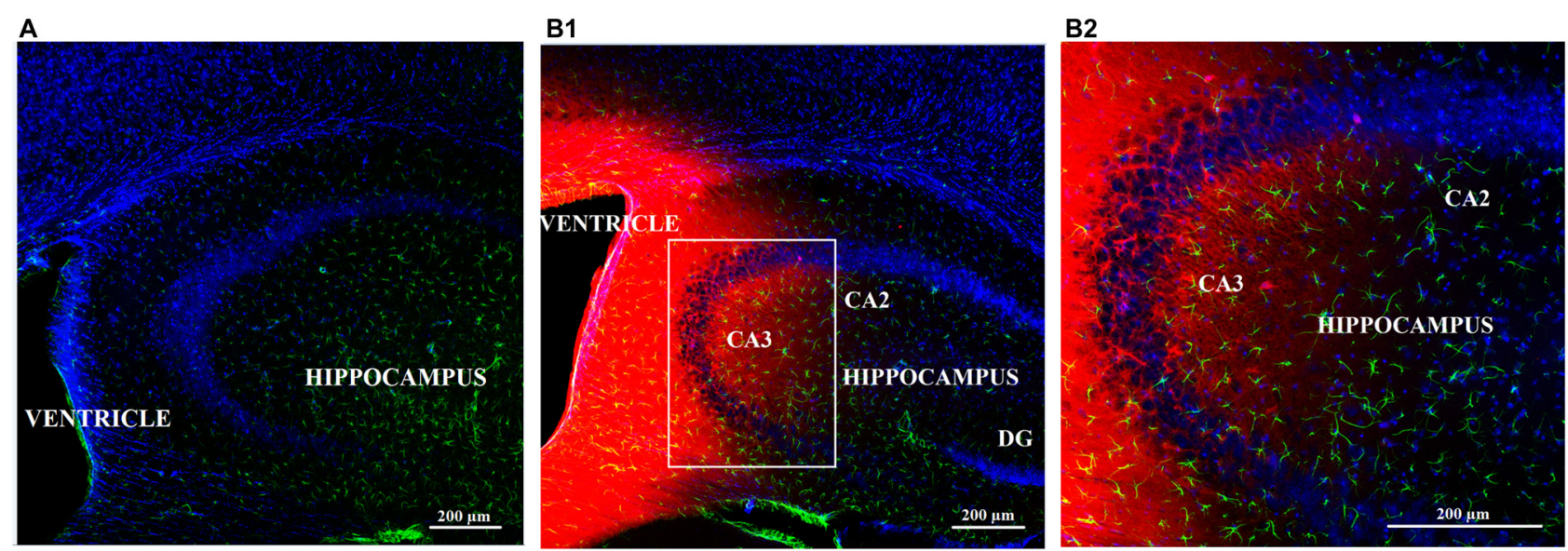

FIGURE 5 | Diffusion of biotin-TAT-Gap19 into the mouse brain 60 min after injection in the left lateral ventricle. Representative vibratome coronal sections (40 $\mathrm{m}$ m thick) through the left ventricle and hippocampus of mice injected with (A) vehicle (PBS) or (B1,2) biotin-TAT-Gap19. Sixty minutes after injection, the brain displays clear biotin immunoreactivity (red) around the ventricle and in the hippocampus compared to mice that received PBS only. (B1) Area in white box is enlarged in the right box of the panel (B2). Sections were double-labeled with anti-GFAP antibody to visualize astrocytes (green) and nuclei were counterstained with DAPI (blue). (Magnification: (A,B1) 10× zoom 0.6 and (B1) 10× zoom 1.3). 
pericytes and endothelial cells; Chew et al., 2010), the observed effect is most likely attributed to astrocytic $\mathrm{Cx} 43 \mathrm{HCs}$, as $\mathrm{Cx} 43$ is predominantly and abundantly found in astrocytes and it is known that gliotransmission through astrocytes is pivotal for learning and (spatial) memory (Hassanpoor et al., 2014). Undoubtedly, astrocytes exert significant effects on synaptic activity and hippocampal neuronal output by ensheathing synapses of these neurons and by releasing gliotransmitters, which have diverse effects on adjacent neurons including place and grid cells (Hassanpoor et al., 2014). The contribution of $\mathrm{Cx} 43 \mathrm{HCs}$ on activated microglia and endothelial cells is most likely negligible, because $\mathrm{Cx} 43$ expression on these cell-types is correlated to brain injury and inflammation (Orellana et al., 2009). Our present experiments were however performed in healthy mice, despite the surgical procedure. Indeed, a recent study by Seyer et al. (2016) demonstrated that spatial working memory was unaffected 1 week after the implantation of an indwelling cannula into the lateral ventricle of rats. On day 6-post surgery, there was no difference in spontaneous alternation score, nor in number of arm entries, assessed in a plus maze. This finding was supported by the relatively small amount of neuronal cell death, limited spread of immune cell activation from the cannulation site and the lack of inflammatory gene upregulation in any area. The authors conclude that there is a very localized inflammatory response that does not extend beyond the immediate site of cannulation. The lack of cognitive deficit due to i.c.v. cannulation might be explained by the fact that the cannula does not descend deep into the brain. By this procedure, only the motor/somatosensory cortex is damaged and these areas are not specifically associated with memory (Seyer et al., 2016). Complementary, we could not find an influence of cannulation on spatial short-term memory as there was no difference in time spent in the novel arm between naïve NMRI mice (without i.c.v cannula) receiving an i.p. injection of $0.9 \%$ $\mathrm{NaCl}$ and i.c.v. cannulated NMRI mice receiving PBS i.c.v (on day 7-post surgery; data not shown). Additionally, studies demonstrating a clear role for acute modulation of endothelial cells or pericytes in spatial memory are still lacking. Therefore, the observed effect is most likely attributed to astrocytic Cx43 HCs.

In summary, our results complement previous studies and indicate for the first time that $\mathrm{Cx} 43 \mathrm{HC}$ inhibition impairs hippocampal short-term spatial memory. However, the results are still preliminary and further research is indicated to fully elucidate the regulatory pathway of $\mathrm{Cx} 43 \mathrm{HCs}$. For instance, the

\section{REFERENCES}

Abbasi, E., Nassiri-Asl, M., Sheikhi, M., and Shafiee, M. (2013). Effects of vitexin on scopolamine-induced memory impairments in rats. Chin. J. Physiol. 56, 184-189. doi: 10.4077/CJP.2013.BAB123

Aben, B., Stapert, S., and Bloklan, A. (2012). About the discrimination between working memory and short-term memory. Front. Psychol. 3:301. doi: 10. 3389/fpsyg.2012.00301

Abudara, V., Bechberger, J., Freitas-Andrade, M., De Bock, M., Wang, N., Bultynck, G., et al. (2014). The connexin43 mimetic peptide Gap19 inhibits hemichannels without altering gap junctional communication in results don't necessarily mean that gliotransmitters are released directly through $\mathrm{Cx} 43 \mathrm{HCs}$. Open HCs can facilitate $\mathrm{Ca}^{2+}$ entry (Schalper et al., 2010) that in turn could trigger $\mathrm{Ca}^{2+}$-dependent exocytosis of gliotransmitters indirectly (Martineau et al., 2014). Indeed, the exocytotic secretory machinery (e.g., soluble N-ethyl maleimide-sensitive fusion protein attachment protein receptor (SNARE) complex) is present in astrocytes (Hamilton, 2010) and astrocytic $\mathrm{Ca}^{2+}$-dependent exocytosis of glutamate, $\mathrm{D}$-serine and ATP has been described in literature (Harada et al., 2016). Additionally, it cannot be excluded that alternative $\mathrm{Ca}^{2+}$. dependent release mechanisms are involved such as release through volume-regulated anion channels (Hamilton, 2010). Additionally, changes of intracellular $\mathrm{Ca}^{2+}$ can modulate other trafficking events in the constitutive secretion and recycling pathways and an increase in $\mathrm{Ca}^{2+}$ could release transmitters from astrocytes by promoting the insertion into the plasma membrane of proteins which mediate non-exocytotic release (Hamilton, 2010).

\section{AUTHOR CONTRIBUTIONS}

LW contributed to the design of the study and performed all the experiments. She acquired the data, performed the data analyses and interpreted the results. LW wrote the draft of the manuscript and created the artwork. MV contributed to the interpretation of the results. GA and DDB helped with optimizing the immunohistochemistry protocol. LL contributed to the conception of the study, the interpretation of data and the artwork. IJS supervised the project. She contributed to the conception and design of the study and the interpretation of the data. All authors revised the manuscript and gave final approval of the version to be submitted and any revised version. All authors agree to be accountable for all aspects of the work in ensuring that questions related to the accuracy of integrity of any part of the work are appropriately investigated and resolved.

\section{ACKNOWLEDGMENTS}

We acknowledge Thomas Demuyser for helping with the artwork and Eddy Himpe for obtaining the confocal microscope images. LW, GA, DDB and IJS were financially supported by the Vrije Universiteit Brussel (OZR2102), MV was supported by the European Research Council (ERC Starting Grant 335476) and LL by the Fund for scientific Research Flanders (FWO Vlaanderen), Belgium (Grant nos G.0298.11, G.0571.12, G.0A54.13 and G.0320.15).

astrocytes. Front. Cell. Neurosci. 8:306. doi: 10.3389/fncel.2014 00306

Ben Achour, S., and Pascual, O. (2010). Glia: the many ways to modulate synaptic plasticity. Neurochem. Int. 57, 440-445. doi: 10.1016/j.neuint.2010.02.013

Busquet, P., Capurro, V., Cavalli, A., Piomelli, D., Reggiani, A., and Bertorelli, R. (2012). Synergistic effects of galantamine and memantine in attenuating scopolamine-induced amnesia in mice. J. Pharmacol. Sci. 120, 305-309. doi: 10. 1254/jphs.12166sc

Chandrasekhar, A., and Bera, A. K. (2012). Hemichannels: permeants and their effect on development, physiology and death. Cell Biochem. Funct. 30, 89-100. doi: $10.1002 / \mathrm{cbf} .2794$ 
Cheung, G., Chever, O., and Rouach, N. (2014). Connexons and pannexons: newcomers in neurophysiology. Front. Cell. Neurosci. 8:348. doi: 10.3389/fncel. 2014.00348

Chever, O., Lee, C. Y., and Rouach, N. (2014a). Astroglial connexin43 hemichannels tune basal excitatory synaptic transmission. J. Neurosci. 34, 11228-11232. doi: 10.1523/JNEUROSCI.0015-14.2014

Chever, O., Pannasch, U., Ezan, P., and Rouach, N. (2014b). Astroglial connexin 43 sustains glutamatergic synaptic efficacy. Philos. Trans. R. Soc. Lond. B Biol. Sci. 369:20130596. doi: 10.1098/rstb.2013.0596

Chew, S. S. L., Johnson, C. S., Green, C. R., and Danesh-Meyer, H. V. (2010). Role of connexin43 in central nervous system injury. Exp. Neurol. 225, 250-261. doi: 10.1016/j.expneurol.2010.07.014

Contreras, J. E., Saéz, J. C., Bukauskas, F. F., and Bennett, M. V. (2003). Gating and regulation of connexin 43 (Cx43) hemichannels. Proc. Natl. Acad. Sci. U S A 100, 11388-11393. doi: 10.1073/pnas.1434298100

Contreras, J. E., Sánchez, H. A., Véliz, L. P., Bukauskas, F. F., Bennett, M. V. L., and Sáez, J. C. (2004). Role of connexin-based gap junction channels and hemichannels in ischemia-induced cell death in nervous tissue. Brain Res. Rev. 47, 290-303. doi: 10.1016/j.brainresrev.2004. 08.002

Cowan, N. (2008). What are the differences between long-term, short-term and working memory? Prog. Brain Res. 169, 323-338. doi: 10.1016/S00796123(07)00020-9

De Bock, M., Decrock, E., Wang, N., Bol, M., Vinken, M., Bultynck, G., et al. (2014). The dual face of connexin-based astroglial $\mathrm{Ca}^{2+}$ communication: a key player in brain physiology and a prime target in pathology. Biochim. Biophys Acta 1843, 2211-2232. doi: 10.1016/j.bbamcr.2014.04.016

de Bruin, N., and Pouzet, B. (2006). Beneficial effects of galantamine on performance in the object recognition task in Swiss mice: deficits induced by scopolamine and by prolonging the retention interval. Pharmacol. Biochem. Behav. 85, 253-260. doi: 10.1016/j.pbb.2006.08.007

De Bundel, D., Schallier, A., Loyens, E., Fernando, R., Miyashita, H., Van Liefferinge, J., et al. (2011). Loss of system $\mathrm{x}_{\mathrm{C}^{-}}$- does not induce oxidative stress but decreases extracellular glutamate in hippocampus and influences spatial working memory and limbic seizure susceptibility. J. Neurosci. 31, 5792-5803. doi: 10.1523/JNEUROSCI.5465-10.2011

De Vuyst, E., Wang, N., Decrock, E., De Bock, M., Vinken, M., Van Moorhem, M., et al. (2009). $\mathrm{Ca}^{2+}$ regulation of connexin 43 hemichannels in C6 glioma and glial cells. Cell Calcium 46, 176-187. doi: 10.1016/j.ceca.2009.07.002

Giaume, C., Leybaert, L., Naus, C. C., and Sáez, J. C. (2013). Connexin and pannexin hemichannels in brain glial cells: properties, pharmacology and roles. Front. Pharmacol. 4:88. doi: 10.3389/fphar.2013.00088

Hamilton, N. B. (2010). Do astrocytes really exocytose neurotransmitters? Nat. Rev. Neurosci. 11, 227-238. doi: 10.1038/nrn2803

Harada, K., Kamiya, T., and Tsuboi, T. (2016). Gliotransmitter release from astrocytes: functional, developmental, and pathological implications in the brain. Front. Neurosci. 9:499. doi: 10.3389/fnins.2015.00499

Hassanpoor, H., Fallah, A., and Raza, M. (2014). Mechanisms of hippocampal astrocytes mediation of spatial memory and theta rhythm by gliotransmitters and growth factors. Cell Biol. Int. 38, 1355-1366. doi: 10.1002/cbin.10326

Iyyathurai, J., D’Hondt, C., Wang, N., De Bock, M., Himpens, B., Retamal, M. A., et al. (2013). Peptides and peptide-derived molecules targeting the intracellular domains of Cx43: gap junctions versus hemichannels. Neuropharmacology 75, 491-505. doi: 10.1016/j.neuropharm.2013.04.050

Johanson, C. E., Duncan, J. A. 3rd, Klinge, P. M., Brinker, T., Stopa, E. G., and Silverberg, G. D. (2008). Multiplicity of cerebrospinal fluid functions: new challenges in health and disease. Cerebrospinal Fluid Res. 5:10. doi: 10. 1186/1743-8454-5-10

Kilkenny, C., Browne, .J., Cuthill, I. C., Emerson, M., and Altman, D. G. (2010). Improving bioscience research reporting: the ARRIVE guidelines for reporting animal research. PLoS Biol. 8:e1000412. doi: 10.1371/journal.pbio.1000412

Kwon, S. H., Lee, H. K., Kim, J. A., Hong, S. I., Kim, H. C., Jo, T. H., et al. (2010). Neuroprotective effects of chlorogenic acid on scopolamine-induced amnesia via anti-acetylcholinesterase and anti-oxidative activities in mice. Eur. J. Pharmacol. 649, 210-217. doi: 10.1016/j.ejphar.2010.09.001

Lee, I., and Kesner, R. P. (2003). Time-dependent relationship between the dorsal hippocampus and the prefrontal cortex in spatial memory. J. Neurosci. 23, 1517-1523.
Martineau, M., Parpura, V., and Mothet, J.-P. (2014). Cell-type specific mechanisms of D-serine uptake and release in the brain. Front. Synaptic Neurosci. 6:12. doi: 10.3389/fnsyn.2014.00012

Mathur, A., Shandarin, A., LaViolette, S. R., Parker, J., and Yeomans, J. S. (1997). Locomotion and stereotypy induced by scopolamine: contributions of muscarinic receptors near the pedunculopontine tegmental nucleus. Brain Res. 775, 144-155. doi: 10.1016/s0006-8993(97)00928-1

Montero, T. D., and Orellana, J. A. (2015). Hemichannels: new pathways for gliotransmitter release. Neuroscience 286, 45-59. doi: 10.1016/j.neuroscience. 2014.11.048

Moraga-Amaro, R., Jerez-Baraona, J. M., Simon, F., and Stehberg, J. (2014). Role of astrocytes in memory and psychiatric disorders. J. Physiol. Paris 108, 240-251. doi: 10.1016/j.jphysparis.2014.08.005

Moser, M.-B., Rowland, D. C., and Moser, E. I. (2015). Place cells, grid cells and memory. Cold Spring Harb. Perspect. Biol. 7:a021808. doi: 10.1101/cshperspect. a021808

Nagy, J. I., and Rash, J. E. (2000). Connexins and gap junctions of astrocytes and oligodendrocytes in the CNS. Brain Res. Rev. 32, 29-44. doi: 10.1016/s01650173(99)00066-1

Naus, C. C. G., Bechberger, J. F., Caveney, S., and Wilson, J. X. (1991). Expression of gap junction genes in astrocytes and C6 glioma cells. Neurosci. Lett. 126, 33-36. doi: 10.1016/0304-3940(91)90364-y

Ohba, T., Yoshino, Y., Ishisaka, M., Abe, N., Tsuruma, K., Shimazawa, M., et al. (2015). Japanese Huperzia serrata extract and the constituent, huperzine A, ameliorate the scopolamine-induced cognitive impairment in mice. Biosci. Biotechnol. Biochem. 79, 1838-1844. doi: 10.1080/09168451.2015.10 52773

Omasits, U., Ahrens, C. H., Müller, S., and Wollscheid, B. (2014). Protter: interactive protein feature visualizationd and integration with experimental proteomic data. Bioinformatics 30, 884-886. doi: 10 1093/bioinformatics/btt607

Orellana, J. A., Saéz, P. J., Shoji, K. F., Schalper, K. A., Palacios-Prado, N., Velarde, V., et al. (2009). Modulation of brain hemichannels and gap junction channels by pro-inflammatory agents and their possible role in neurodegeneration. Antioxid. Redox Signal. 11, 369-399. doi: 10.1089/ars.2008. 2130

Pannasch, U., and Rouach, N. (2013). Emerging role for astroglial networks in information processing: from synapse to behavior. Trends Neurosci. 36, 405-417. doi: 10.1016/j.tins.2013.04.004

Pardridge, W. M. (1991). Transnasal and Intraventricular Delivery. Peptide Drug Delivery to the Brain. Michigan: Raven Press.

Paxinos, G., and Franklin, K. B. (2004). The Mouse Brain in Stereotaxic Coordinates. Compact Second Edition. San Diego, CA: Elsevier Academic Press.

Schalper, K. A., Sánchez, H. A., Lee, S. C., Altenberg, G. A., Nathanson, M. H., and Sáez, J. C. (2010). Connexin 43 hemichannels mediate the $\mathrm{Ca}^{2+}$ influx induced by extracellular alkalinization. Am. J. Physiol. Cell Physiol. 299, C1504-C1515. doi: 10.1152/ajpcell.00015.2010

Seyer, B., Pham, V., Albiston, A. L., and Chai, S. Y. (2016). Cannula implantation into the lateral ventricle does not adversely affect recognition or spatial working memory. Neurosci. Lett. 628, 171-178. doi: 10.1016/j.neulet.2016. 06.034

Sharma, S., Rakoczy, S., and Brown-Borg, H. (2010). Assessment of spatial memory in mice. Life Sci. 87, 521-536. doi: 10.1016/j.lfs.2010. 09.004

Shipton, O. A., El-Gaby, M., Apergis-Schoute, J., Deisseroth, K., Bannerman, D. M., Paulsen, O., et al. (2014). Left-right dissociation of hippocampal memory processes in mice. Proc. Natl. Acad. Sci. U S A 111, 15238-15243. doi: 10. 1073/pnas.1405648111

Stehberg, J., Moraga-Amaro, R., Salazar, C., Becerra, A., Echeverría, C., Orellana, J. A., et al. (2012). Release of gliotransmitters through astroglial connexin 43 hemichannels is necessary for fear memory consolidation in the basolateral amygdala. FASEB J. 26, 3649-3657. doi: 10.1096/fj.11198416

Tabari, S. S., Babri, S., Mirzaie, F., Farajdokht, F., and Mohaddes, G. (2016). Enduring amnesia induced by ICV scopolamine is reversed by sesame oil in male rats. Acta Cir. Bras. 31, 520-526. doi: 10.1590/s0102865020160080000004 
Torres, A., Wang, F., Xu, Q., Fujita, T., Dorbrowolski, R., Willecke, K., et al. (2012). Extracellular $\mathrm{Ca}^{2+}$ acts as a mediator of communication from neurons to glia. Sci. Signal. 5:ra8. doi: 10.1126/scisignal.2002160

Wall, P. M., and Messier, C. (2002). Infralimbic kappa opioid and muscarinic $\mathrm{M} 1$ receptor interactions in the concurrent modulation of anxiety and memory. Psychopharmacology (Berl) 160, 233-244. doi: 10.1007/s00213-001-0979-9

Wang, N., De Bock, M., Decrock, E., Bol, M., Gadicherla, A., Bultynck, G., et al. (2013a). Connexin targeting peptides as inhibitors of voltage- and intracellular $\mathrm{Ca}^{2+}$-triggered Cx43 hemichannel opening. Neuropharmacology 75, 506-516. doi: 10.1016/j.neuropharm.2013.08.021

Wang, N., De Vuyst, E., Ponsaerts, R., Boengler, K., Palacios-Prado, N., Wauman, J., et al. (2013b). Selective inhibition of $\mathrm{Cx} 43$ hemichannels by Gap19 and its impact on myocardial ischemia/reperfusion injury. Basic Res. Cardiol. 108:309. doi: 10.1007/s00395-012-0309-X

Wei, H., Deng, F., Chen, Y., Qin, Y., Hao, Y., and Guo, X. (2014). Ultrafine carbon black induces glutamate and ATP release by activating connexin and pannexin hemichannels in cultured astrocytes. Toxicology 323, 32-41. doi: 10.1016/j.tox. 2014.06.005
Winkler, E. A., Bell, R. D., and Zlokovic, B. V. (2011). Central nervous system pericytes in health and disease. Nat. Neurosci. 14, 1398-1405. doi: 10.1038/nn. 2946

Yoon, T., Okada, J., Jung, M. W., and Kim, J. J. (2008). Prefrontal cortex and hippocampus subserve different components of working memory in rats. Learn. Mem. 15, 97-105. doi: 10.1101//m.850808

Conflict of Interest Statement: The authors declare that the research was conducted in the absence of any commercial or financial relationships that could be construed as a potential conflict of interest.

Copyright (c) 2016 Walrave, Vinken, Albertini, De Bundel, Leybaert and Smolders. This is an open-access article distributed under the terms of the Creative Commons Attribution License (CC BY). The use, distribution and reproduction in other forums is permitted, provided the original author(s) or licensor are credited and that the original publication in this journal is cited, in accordance with accepted academic practice. No use, distribution or reproduction is permitted which does not comply with these terms. 